Article

\title{
Solution to the Time-Dependent Coupled Harmonic Oscillators Hamiltonian with Arbitrary Interactions
}

\author{
Alejandro R. Urzúa ${ }^{1}$ (D) Irán Ramos-Prieto ${ }^{2}$, , Manuel Fernández-Guasti ${ }^{3}$ (i) \\ and Héctor M. Moya-Cessa ${ }^{1, *(1)}$ \\ 1 Instituto Nacional de Astrofísica, Óptica y Electrónica, Calle Luis Enrique Erro No. 1, \\ 72840 Sta. María Tonantzintla, Puebla, Mexico \\ 2 Instituto de Ciencias Físicas, Universidad Nacional Autónoma de México, Apartado Postal 48-3, \\ 62251 Cuernavaca, Morelos, Mexico \\ 3 Departamento de Física, CBI, Universidad Autónoma Metropolitana Iztapalapa, Apartado Postal 55-534, \\ 09340 México D.F., Mexico \\ * Correspondence: hmmc@inaoep.mx; Tel.: +52-222-266-3100
}

Received: 10 June 2019; Accepted: 17 July 2019; Published: 22 July 2019

\begin{abstract}
We show that by using the quantum orthogonal functions invariant, we found a solution to coupled time-dependent harmonic oscillators where all the time-dependent frequencies are arbitrary. This system may be found in many applications such as nonlinear and quantum physics, biophysics, molecular chemistry, and cosmology. We solve the time-dependent coupled harmonic oscillators by transforming the Hamiltonian of the interaction using a set of unitary operators. In passing, we show that $N$ time-dependent and coupled oscillators have a generalized orthogonal functions invariant from which we can write a Ermakov-Lewis invariant.
\end{abstract}

Keywords: time-dependent coupled oscillators; invariants; harmonic oscillators

\section{Introduction}

The harmonic oscillator is one of the most studied systems in physics. Because for any potential, its minimums may always be approximated as quadratic functions, the harmonic oscillator is a cornerstone of physics, in general and quantum mechanics in particular. Time-dependent harmonic oscillators arise in quantum mechanical systems such as optical trapping of different objects like atoms and molecules [1, 2], living cells, including viruses and bacteria [3,4]. Extensions to coupled time-dependent harmonic oscillators arise in several quantum mechanical problems, such as ion-laser interactions [1], quantized fields propagating through dielectric media [5], shortcuts to adiabaticity [6,7], the Casimir effect [8,9], as a toy model to study aspects of the final stages of black-hole evaporation [10], and their Schmidt modes in the study of quantum entanglement [11].

The existence of invariants for time-dependent Hamiltonians in mechanical systems has attracted considerable interest over the years [12]. Such constants of motion are of central importance in the study of dynamical systems. A variety of methods to obtain invariants of systems with one degree of freedom have been developed [13-15]. In particular, the time-dependent harmonic oscillator (TDHO) has received much attention because of its applications in several areas of physics [16]. Among the many procedures developed to obtain invariants, a derivation for the classical TDHO has been presented, which leads directly to the orthogonal functions invariant or to the Lewis invariant [17]. More recently, invariant 
techniques have been put forward to study approximate solutions to time-dependent problems involving trapped particles [18].

The extension of the theory of invariants to the quantum realm has evolved in at least two directions. On the one hand, the one-dimensional time-independent Schrödinger equation is equivalent to the TDHO equation. The translation between equations requires a constant shift of the potential $V(x)$ with the appropriate scaling for the initially time-dependent parameter $\Omega^{2}(t) \rightarrow 2 m(\mathcal{E}-V(x))$ as well as an exchange of temporal and spatial variables. The results obtained in the classical invariant theory are thus applicable for spatially arbitrary time-independent potentials in stationary one-dimensional quantum theory. Using these techniques, how to define coherent states associated to the TDHO [19] and amplitude-phase invariants [20] has been shown. On the other hand, quantum mechanical expressions of the classical invariant operators may be used to obtain exact solutions to the time-dependent Schrödinger equation, such that the classical Hamiltonian is translated into a quantum Hamiltonian by considering the canonical coordinate and momentum as time-independent operators obeying the commutator $[\hat{q}, \hat{p}]=i$ (we set $\hbar=1$ throughout the manuscript). The quantum treatment then becomes a $1+1$ dimensional problem where the wave function depends on a spatial as well as temporal variable. Exact invariants have been derived to tackle a limited class of potentials [21]. The most relevant cases are the quadratic spatial dependence that leads to the quantum mechanical time-dependent harmonic oscillator (QM-TDHO) and the linear potential [22].

The simple extension to two coupled time-dependent harmonic oscillators has been considered and its solution presented by Macedo and Guedes for a very limited case of time-dependent functions [23]. Duncan and del Campo [7] solved the time- dependent coupled harmonic oscillator by considering the time-dependent coefficients as being, effectively, time independent. The Ermakov-Lewis invariant has also been proposed for systems of coupled harmonic oscillators [24].

The QM-TDHO has been solved under various scenarios, such as time-dependent mass $[25,26]$ and damping [27]. Several techniques have been used to solve the corresponding time-dependent Schrödinger equation, such as the time-space re-scaling, transformation methods, and the time-dependent invariant method [28]. The constant of motion that has been invoked in the latter procedure is the well-known Lewis invariant [29]. Applications of invariant methods have been used in adiabatic regimes [30] for the control of quantum noise [31] and the propagation of light in waveguide arrays [32-35].

The main purpose of the present contribution is to show a method to solve the Schrödinger equation for a pair of position coupled time-dependent harmonic oscillators when all the time-dependent functions involved are completely arbitrary, i.e., they are not related to each other. Although the main content of this contribution is the solution of the coupled time-dependent harmonic oscillators, we take the opportunity to translate the invariant recently introduced by Thylwe [24] for $N$ classical coupled harmonic oscillators to the quantum realm.

Summarily, to the best of our knowledge, we present here for the first time, the solution to the problem of two coupled harmonic oscillators with completely arbitrary time-dependent parameters. Former solutions were given only for time-dependent parameters that were related among themselves [23] or were considered constant by using approximate adiabatic techniques [7].

In the following sections, through a series of unitary transformations, some of them time-dependent, we manage to take the Hamiltonian for the two coupled harmonic oscillators to an integrable form. We do so by generalizing some operator methods for the single time-dependent quantum harmonic oscillator introduced in reference [36]. 


\section{Ermakov-Lewis Invariant for $N$ Coupled Time-Dependent Harmonic Oscillators}

Consider the system of differential equations for $N$ time-dependent coupled classical oscillators

$$
\begin{aligned}
\ddot{u}_{1}+\Omega_{1}^{2}(t) u_{1} & =-\eta_{12}(t) u_{2} \\
\ddot{u}_{2}+\Omega_{2}^{2}(t) u_{2} & =-\eta_{12}(t) u_{1}-\eta_{23}(t) u_{3} \\
\ddot{u}_{3}+\Omega_{3}^{2}(t) u_{3} & =-\eta_{23}(t) u_{2}-\eta_{34}(t) u_{4} \\
\vdots & \\
\ddot{u}_{N}+\Omega_{N}^{2}(t) u_{N} & =-\eta_{N-1 N}(t) u_{N-1},
\end{aligned}
$$

with the associated quantum Hamiltonian

$$
\begin{aligned}
\hat{H}_{N}(t) & =\frac{1}{2}\left(\hat{p}_{1}^{2}+\Omega_{1}^{2}(t) \hat{x}_{1}^{2}\right)+\frac{1}{2}\left(\hat{p}_{2}^{2}+\Omega_{2}^{2}(t) \hat{x}_{2}^{2}\right)+\cdots+\frac{1}{2}\left(\hat{p}_{N}^{2}+\Omega_{N}^{2}(t) \hat{x}_{N}^{2}\right) \\
& +\eta_{12}(t) \hat{x}_{1} \hat{x}_{2}+\eta_{23}(t) \hat{x}_{2} \hat{x}_{3}+\cdots+\eta_{N-1 N}(t) \hat{x}_{N-1} \hat{x}_{N} .
\end{aligned}
$$

A single time-dependent harmonic oscillator has quantum orthogonal functions invariant [36],

$$
\hat{G}_{1}=u_{1}(t) \hat{p}_{1}-\dot{u}_{1}(t) \hat{x}_{1}
$$

where $u_{1}(t)$ is the solution of the equation $\ddot{u}_{1}+\Omega_{1}^{2}(t) u_{1}=0$. This invariant may be generalized to $N$ coupled time-dependent harmonic oscillators,

$$
\hat{G}_{N}=u_{1}(t) \hat{p}_{1}-\dot{u}_{1}(t) \hat{x}_{1}+u_{2}(t) \hat{p}_{2}-\dot{u}_{2}(t) \hat{x}_{2}+\cdots+u_{N}(t) \hat{p}_{N}-\dot{u}_{N}(t) \hat{x}_{N},
$$

where the $u^{\prime}$ s satisfy (1), such that

$$
\frac{\partial \hat{G}_{N}}{\partial t}=\dot{u}_{1}(t) \hat{p}_{1}-\ddot{u}_{1}(t) \hat{x}_{1}+\dot{u}_{2}(t) \hat{p}_{2}-\ddot{u}_{2}(t) \hat{x}_{2}+\cdots+\dot{u}_{N}(t) \hat{p}_{N}-\ddot{u}_{N}(t) \hat{x}_{N} .
$$

On the other hand, the commutator between $\hat{G}_{N}$ and $\hat{H}_{N}$, is

$$
\begin{aligned}
i\left[\hat{G}_{N}, \hat{H}_{N}\right] & =\Omega_{1}^{2}(t) u_{1} \hat{x}_{1}+\dot{u}_{1}(t) \hat{p}_{1}+\eta_{12} u_{1} \hat{x}_{2} \\
& +\Omega_{2}^{2}(t) u_{2} \hat{x}_{2}+\dot{u}_{2}(t) \hat{p}_{2}+\eta_{12} u_{2} \hat{x}_{1}+\eta_{23} u_{2} \hat{x}_{3} \\
& \vdots \\
& +\Omega_{N}^{2}(t) u_{N} \hat{x}_{N}+\dot{u}_{N}(t) \hat{p}_{N}+\eta_{N-1 N} u_{N} \hat{x}_{N-1},
\end{aligned}
$$

and by subtracting the above equations, we obtain

$$
\begin{aligned}
\frac{d \hat{G}_{N}}{d t}=\frac{\partial \hat{G}_{N}}{\partial t}-i\left[\hat{G}_{N}, \hat{H}_{N}\right] & =-\left[\Omega_{1}^{2}(t) u_{1}+\ddot{u}_{1}\right] \hat{x}_{1}-\eta_{12} u_{1} \hat{x}_{2} \\
& -\left[\Omega_{2}^{2}(t) u_{2}+\ddot{u}_{2}\right] \hat{x}_{2}-\eta_{12} u_{2} \hat{x}_{1}-\eta_{23} u_{2} \hat{x}_{3} \\
& \vdots \\
& -\left[\Omega_{N}^{2}(t) u_{N}+\ddot{u}_{N}\right] \hat{x}_{N}-\eta_{N-1 N} u_{N} \hat{x}_{N-1} .
\end{aligned}
$$


Rearranging the above expression:

$$
\begin{aligned}
\frac{d \hat{G}_{N}}{d t}=\frac{\partial \hat{G}_{N}}{\partial t}-i\left[\hat{G}_{N}, \hat{H}_{N}\right] & =-\left[\Omega_{1}^{2}(t) u_{1}+\ddot{u}_{1}+\eta_{12}(t) u_{2}\right] \hat{x}_{1} \\
& -\left[\Omega_{2}^{2}(t) u_{2}+\ddot{u}_{2}+\eta_{12}(t) u_{1}+\eta_{23}(t) u_{3}\right] \hat{x}_{2} \\
& \vdots \\
& -\left[\Omega_{N}^{2}(t) u_{N}+\ddot{u}_{N}+\eta_{N-1 N}(t) u_{N-1}\right] \hat{x}_{N}
\end{aligned}
$$

so that (1) gives zero shows that $\hat{G}_{N}$ is indeed an invariant.

By writing, for a single harmonic oscillator, the amplitude $u_{1}=\rho_{1} \exp \left(-i \int d t / \rho_{1}^{2}\right)$, where $\rho_{1}$ obeys the Ermakov equation [36]

$$
\ddot{\rho}_{1}+\Omega_{1}^{2}(t) \rho_{1}=\frac{1}{\rho_{1}^{3}},
$$

the so-called Ermakov-Lewis invariant may be obtained from $\hat{G}_{1}$ as

$$
\hat{I}_{1}=\hat{G}_{1} \hat{G}_{1}^{+}=\frac{1}{2}\left(\frac{\hat{x}_{1}^{2}}{\rho_{1}^{2}}+\left(\rho_{1} \hat{p}_{1}-\dot{\rho}_{1} \hat{x}_{1}\right)^{2}\right),
$$

so that we may write the Ermakov-Lewis invariant for the $N$ coupled time-dependent harmonic oscillators as

$$
\hat{I}_{N}=\hat{G}_{N} \hat{G}_{N}^{+}
$$

\section{The Classical Invariant}

Quantization procedures allow us to associate operators to observables. By doing the opposite procedure, i.e., associating c-numbers to momentum and position operators, $\hat{p} \rightarrow \dot{v}$ and $\hat{x} \rightarrow v$, respectively, we may find the classical invariant from (4)

$$
G_{N}=u_{1}(t) \dot{v}_{1}(t)-\dot{u}_{1}(t) v_{1}(t)+u_{2}(t) \dot{v}_{2}(t)-\dot{u}_{2}(t) v_{2}(t)+\cdots+u_{N}(t) \dot{v}_{N}(t)-\dot{u}_{N}(t) v_{N}(t),
$$

where the $u^{\prime}$ s and $v^{\prime}$ s are linearly independent solutions of (1).

\section{Two Coupled Time-Dependent Harmonic Oscillators}

We consider the time-dependent Hamiltonian for the interacting oscillators (we set the masses as equal to one, because, as Macedo and Guedes showed [23], a simple transformation may be applied that makes the assumption valid)

$$
\hat{H}(t)=\frac{1}{2}\left[\hat{p}_{x}^{2}+\hat{p}_{y}^{2}+\kappa_{x}(t) \hat{x}^{2}+\kappa_{y}(t) \hat{y}^{2}\right]+\kappa(t)(\hat{x}-\hat{y})^{2},
$$

with $\kappa^{\prime}$ s being the time-dependent spring parameters. By setting $\Omega_{x}^{2}(t)=\kappa_{x}(t)+\kappa(t), \Omega_{y}^{2}(t)=\kappa_{y}(t)+$ $\kappa(t)$, and $\eta(t)=-2 \kappa(t)$, we end up with the $\hat{x} \hat{y}$ interaction Hamiltonian

$$
\hat{H}(t)=\frac{1}{2}\left[\hat{p}_{x}^{2}+\hat{p}_{y}^{2}+\Omega_{x}^{2}(t) \hat{x}^{2}+\Omega_{y}^{2}(t) \hat{y}^{2}\right]+\eta(t) \hat{x} \hat{y} .
$$

The classical equations of motion for the above Hamiltonian are

$$
\ddot{u}_{x}+\Omega_{x}^{2}(t) u_{x}=-\eta(t) u_{y}, \quad \ddot{u}_{y}+\Omega_{y}^{2}(t) u_{y}=-\eta(t) u_{x}
$$


where the quantum invariants of each coupled oscillator are [37]

$$
\hat{G}_{x}=\left(u_{x} \hat{p}_{x}-\hat{x} \dot{u}_{x}\right)-\int \eta(t)\left(u_{x} \hat{y}-\hat{x} u_{y}\right) d t
$$

and

$$
\hat{G}_{y}=\left(u_{y} \hat{p}_{y}-\hat{y} \dot{u}_{y}\right)-\int \eta(t)\left(u_{y} \hat{x}-\hat{y} u_{x}\right) d t,
$$

since the total invariant must comply with

$$
\frac{\partial}{\partial t}\left(\hat{G}_{x}+\hat{G}_{y}\right)-i\left[\hat{G}_{x}+\hat{G}_{y}, \hat{H}\right]=0 .
$$

We now consider the transformation [36]

$$
\hat{T}_{u}=e^{i \frac{\ln u_{x}(t)}{2}\left(\hat{x} \hat{p}_{x}+\hat{p}_{x} \hat{x}\right)} e^{-i \frac{\dot{u}_{x}(t)}{2 u_{x}(t)} \hat{x}^{2}} e^{i \frac{\ln u_{y}(t)}{2}\left(\hat{y} \hat{p}_{y}+\hat{p}_{y} \hat{y}\right)} e^{-i \frac{u_{y}(t)}{2 u_{y}(t)} \hat{y}^{2}}
$$

that produces

$$
\begin{aligned}
\hat{T}_{u} \hat{x} \hat{T}_{u}^{\dagger} & =u_{x} \hat{x} \\
\hat{T}_{u} \hat{y} \hat{T}_{u}^{\dagger} & =u_{y} \hat{y} \\
\hat{T}_{u} \hat{p}_{x} \hat{T}_{u}^{\dagger} & =\frac{\hat{p}_{x}}{u_{x}}+\dot{u}_{x} \hat{x}, \\
\hat{T}_{u} \hat{p}_{y} \hat{T}_{u}^{\dagger} & =\frac{\hat{p}_{y}}{u_{y}}+\dot{u}_{y} \hat{y},
\end{aligned}
$$

where $u$ is the solution to the TDHO equation (15).

If we transform the wave function with the transformation above, i.e.,

$$
\left|\phi_{u}(t)\right\rangle=\hat{T}_{u}|\psi(t)\rangle,
$$

the Schrödinger equation

$$
i \frac{\partial|\psi(t)\rangle}{\partial t}=\hat{H}(t)|\psi(t)\rangle
$$

has to be rewritten. In order to do it, substitution in Equation (22) leads to

$$
i\left(\hat{T}_{u}^{\dagger} \frac{\partial\left|\phi_{u}(t)\right\rangle}{\partial t}+\frac{\partial \hat{T}_{u}^{\dagger}}{\partial t}\left|\phi_{u}(t)\right\rangle\right)=\hat{H}(t) \hat{T}_{u}^{\dagger}\left|\phi_{u}(t)\right\rangle .
$$

By noting that

$$
\frac{\partial \hat{T}_{u}^{+}}{\partial t}=\frac{i}{2} \hat{T}_{u}^{+}\left[\left(u_{x} \ddot{u}_{x}-\dot{u}_{x}^{2}\right) \hat{x}^{2}-\frac{\dot{u}_{x}}{u_{x}}\left(\hat{p}_{x} \hat{x}+\hat{x} \hat{p}_{x}\right)+\left(u_{y} \ddot{u}_{y}-\dot{u}_{y}^{2}\right) \hat{y}^{2}-\frac{\dot{u}_{y}}{u_{y}}\left(\hat{p}_{y} \hat{y}+\hat{y} \hat{p}_{y}\right)\right],
$$

or

$$
i \frac{\partial\left|\phi_{u}(t)\right\rangle}{\partial t}=\frac{1}{2}\left[\frac{\hat{p}_{x}^{2}}{u_{x}^{2}}+\left(\Omega_{x}^{2} u_{x}+\ddot{u}_{x}\right) u_{x} \hat{x}^{2}+\frac{\hat{p}_{y}^{2}}{u_{y}^{2}}+\left(\Omega_{y}^{2} u_{y}+\ddot{u}_{y}\right) u_{y} \hat{y}^{2}+\eta(t) u_{x} u_{y} \hat{x} \hat{y}\right]\left|\phi_{u}(t)\right\rangle,
$$


from (15), we may rewrite the Schrödinger equation as

$$
i \frac{\partial\left|\phi_{u}(t)\right\rangle}{\partial t}=\frac{1}{2}\left[\frac{\hat{p}_{x}^{2}}{u_{x}^{2}}+\frac{\hat{p}_{y}^{2}}{u_{y}^{2}}-\eta(t) u_{x} u_{y}(\hat{x}-\hat{y})^{2}\right]\left|\phi_{u}(t)\right\rangle .
$$

We now perform a second transformation, $\left|\phi_{\theta}\right\rangle=\hat{R}_{\theta}\left|\phi_{u}\right\rangle$, with $\hat{R}_{\theta}=\exp \left[i \theta\left(\hat{x} \hat{p}_{y}-\hat{y} \hat{p}_{x}\right)\right]$

$$
i \frac{\partial\left|\phi_{\theta}\right\rangle}{\partial t}=\hat{H}_{\theta}(t)\left|\phi_{\theta}\right\rangle
$$

such that the different operators are transformed according to

$$
\begin{aligned}
\hat{R}_{\theta} \hat{x} \hat{R}_{\theta}^{+} & =\hat{x} \cos \theta-\hat{y} \sin \theta, \\
\hat{R}_{\theta} \hat{y} \hat{R}_{\theta}^{+} & =\hat{y} \cos \theta+\hat{x} \sin \theta, \\
\hat{R}_{\theta} \hat{p}_{x} \hat{R}_{\theta}^{+} & =\hat{p}_{x} \cos \theta-\hat{p}_{y} \sin \theta, \\
\hat{R}_{\theta} \hat{p}_{y} \hat{R}_{\theta}^{+} & =\hat{p}_{y} \cos \theta+\hat{p}_{x} \sin \theta .
\end{aligned}
$$

By setting $\theta=\pi / 4$ to arrive to the integrable equation

$$
i \frac{\partial\left|\phi_{\theta}(t)\right\rangle}{\partial t}=\left[\frac{\hat{p}_{x}^{2}+\hat{p}_{y}^{2}}{2 \mu(t)}+\frac{\hat{p}_{x} \hat{p}_{y}}{2 v(t)}+\lambda(t) \hat{y}^{2}\right]\left|\phi_{\theta}(t)\right\rangle,
$$

with

$$
\frac{1}{\mu(t)}=\frac{1}{2 u_{x}^{2}}+\frac{1}{2 u_{y}^{2}}, \quad \frac{1}{v(t)}=\frac{1}{u_{y}^{2}}-\frac{1}{u_{x}^{2}}, \quad \lambda(t)=\frac{\eta(t) u_{x} u_{y}}{\sqrt{2}} .
$$

Note that the Hamiltonian in Equation (29) shows that the operators involved in variable $\hat{x}$ commute as they are simple powers of $\hat{p}_{x}$, such that Equation (29) is readily solvable as this operator acts as a $c$-number for the variable $\hat{y}$. Therefore, we have been able to split the Hamiltonian into two; a term that is a free particle in $\hat{x}$ (time-dependent) and a TDHO in $\hat{x}$ with an extra term, damping, proportional to $\hat{p}_{x}$.

In order to convert the equation above to a more familiar form, we transform, with the unitary operator $\hat{D}=\exp \left\{i \alpha(t) \hat{p}_{y}\right\} \exp \{\beta(t) \hat{y}\}$, the above equation, namely $\left|\phi_{D}\right\rangle=\hat{D}\left|\phi_{\theta}\right\rangle$, and we obtain the final and solvable form of the Hamiltonian

$$
i \frac{\partial\left|\phi_{D}(t)\right\rangle}{\partial t}=\left[\frac{\hat{p}_{y}^{2}}{2 \mu(t)}+\lambda(t) \hat{y}^{2}+\frac{\hat{p}_{x}^{2}}{2 \mu(t)}-\frac{\beta \hat{p}_{x}}{2 \nu(t)}+\alpha \dot{\beta}+\lambda \alpha^{2}+\frac{\beta^{2}}{2 \mu}\right]\left|\phi_{D}(t)\right\rangle,
$$

where $\alpha$ and $\beta$ are functions not only of time, but of the momentum operator $\hat{p}_{x}$, and obey the system of differential equations

$$
\dot{\alpha}+\frac{\beta}{\mu}-\frac{\hat{p}_{x}}{2 v}=0, \quad \dot{\beta}-2 \lambda \alpha=0 .
$$

We note that the Hamiltonian in Equation (31) has been separated in two parts: one of them is a time-dependent harmonic oscillator that depends only on $\hat{y}$ and $\hat{p}_{y}$ (and powers of them) and therefore there are Ermakov-Lewis methods to solve it; the other depends only on $\hat{p}_{x}$ (and its powers) and therefore is integrable [36]. In addition, within the framework of shortcuts to adiabaticity [6], Equation (32) provides quick routes to obtain final results of slow adiabatic processes. 
Finally, it is worth mentioning how the different transformations act on wave functions. It is not difficult to show that

$$
\hat{R}_{\theta} \phi(x, y)=\phi\left[\frac{\cos \theta x-\sin \theta y}{\cos ^{2} \theta}, \sin \theta x+\cos \theta y\right],
$$

where $\phi(x, y)$ is an arbitrary, but well behaved, function of $x$ and $y$.

To study the action of the $\hat{R}_{\theta}$ operator over an arbitrary function $F(x, y)$, we make

$$
\hat{R}_{\theta}=\exp \left[i \theta\left(\hat{x} \hat{p}_{y}-\hat{y} \hat{p}_{x}\right)\right]=\hat{T}_{y} \hat{S}_{x y} \hat{T}_{x}
$$

where

$$
\begin{aligned}
\hat{T}_{y} & =\exp \left[i \tan \theta \hat{x} \hat{p}_{y}\right], \\
\hat{S}_{x y} & =\exp \left\{-i \ln [\cos \theta]\left(\hat{x} \hat{p}_{x}-\hat{y} \hat{p}_{y}\right)\right\} \\
\hat{T}_{x} & =\exp \left[-i \tan \theta \hat{y} \hat{p}_{x}\right] .
\end{aligned}
$$

Note that the operator $\hat{S}_{x y}$ is a product of squeeze operators [38-42] in $x$ and $y$. We can prove that as

$$
\hat{T}_{x} F(x)=F[x-y \tan \theta], \quad \hat{T}_{y} G(y)=G[y+x \tan \theta],
$$

and the action of the squeeze operators

$$
\exp \left(\operatorname{ir} \hat{p}_{x} \hat{x}\right) x=\exp (2 r) x, \quad \exp \left(i r \hat{p}_{y} \hat{y}\right) y=\exp (2 r) y .
$$

\section{Conclusions}

We have shown that the quantum invariant for $\mathrm{N}$-coupled time-dependent harmonic oscillators is indeed constant for arbitrary restitutive oscillator time-dependent functions as well as the existence of arbitrary time-dependent coupling between them. We have translated this result into its classical version. In the case of the two oscillators, we have shown how to solve the Hamiltonian for arbitrary functions of time, as formerly it had been solved only when the functions were related in specific ways [23]. We did it by using the orthogonal functions invariant introduced in [36] which allowed us to split the Hamiltonian in such a way that it was left to solve a single time-dependent harmonic oscillator, which is a well-known problem $[23,29]$.

The Ermakov-Lewis invariant for a single oscillator does not involve the time-dependent parameters explicitly. This well-known fact is not fulfilled when each coupled oscillator is considered separately. The quantum invariants $\hat{G}_{x}$ and $\hat{G}_{y}$ given by (16) and (17) involve the coupling variable $\eta(t)$. However, the Ermakov-Lewis invariant of the whole system, in this case, the two coupled oscillators, i.e., $\hat{G}_{x}+\hat{G}_{y}$, no longer involves $\eta(t)$. Therefore, the invariant of the complete system is again explicitly independent of the time-varying parameters. This remark is also evinced for the $\mathrm{N}$-coupled system. The invariant for $N$-coupled oscillators (4), is the Ermakov invariant of the complete system, it is again explicitly independent of any of the time-varying parameters.

We would like to stress that the key ingredient to our solution is the transformation given by Equation (19) [36]. This mapping allowed us to eventually separate the coupled time-dependent Hamiltonian into the form given in Equation (29). This expression is already integrable, as its Hamiltonian has the form of a damped harmonic oscillator, because the operator $\hat{p}_{x}$ and its powers behave as c-numbers in the Hamiltonian of Equation (29).

To the best of our knowledge, this is the first time that the solution for the two coupled harmonic oscillators Hamiltonian, with arbitrary time- dependent parameters, has been obtained. 
Author Contributions: A.R.U. conceived the idea and all the authors developed it. The manuscript was written by all authors, who have read and approved the final manuscript.

Funding: This research received no external funding.

Conflicts of Interest: The authors declare no conflict of interest.

\section{References}

1. Moya-Cessa, H.; Soto-Eguibar, F. Vargas-Martinez, J.M.; Juarez-Amaro, R.; Zuñiga-Segundo, A. Ion-laser interactions: The most complete solution. Phys. Rep. 2012, 513, 229-261. [CrossRef]

2. Cronin, A.D.; Schmiedmayer, J.; Pritchard, D.E. Optics and interferometry with atoms and molecules. Rev. Mod. Phys. 2009, 81, 1051. [CrossRef]

3. Ashkin, A.; Dziedzic, J.M.; Yamane, T. Optical trapping and manipulation of single cells using infrared laser beams. Nature 1987, 330, 769. [CrossRef] [PubMed]

4. Ashkin A.; Dziedzic, J.M. Optical trapping and manipulation of viruses and bacteria. Science 1987, 235, 1517-1520. [CrossRef] [PubMed]

5. Law, C.K. Effective Hamiltonian for the radiation in a cavity with a moving mirror and a time-varying dielectric medium. Phys. Rev. A 1994, 49, 433. [CrossRef] [PubMed]

6. Chen, X.; Ruschhaupt, A.; Schmidt, S.; del Campo, A.; Guéry-Odelin, D.; Muga, J.G. Fast Optimal Frictionless Atom Cooling in Harmonic Traps: Shortcut to Adiabaticity. Phys. Rev. Lett. 2010, 104, 063002. [CrossRef] [PubMed]

7. Duncan, C.W.; del Campo, A. Shortcuts to adiabaticity assisted by counterdiabatic Born-Oppenheimer dynamics. New J. Phys. 2018, 20, 085003. [CrossRef]

8. Dodonov, V.V.; Klimov, A.B. Generation and detection of photons in a cavity with a resonantly oscillating boundary. Phys. Rev. A 1996, 53, 2664. [CrossRef] [PubMed]

9. Román-Ancheyta, R.; Ramos-Prieto, I.; Perez-Leija, A.; Busch, K.; León-Montiel. R.D.J. Dynamical Casimir effect in stochastic systems: Photon harvesting through noise. Phys. Rev. A 2017, 96, 032501. [CrossRef]

10. Kiefer, C.; Marto, J.; Moniz, P.V. Indefinite oscillators and black-hole evaporation. Ann. Phys. 2012, 18, 722-735. [CrossRef]

11. Makarov, D.N. Coupled harmonic oscillators and their quantum entanglement. Phys. Rev. E 2018, 97, 042203. [CrossRef] [PubMed]

12. Bouquet S.; Lewis, V J. A second invariant for one-degree-of-freedom, time-dependent Hamiltonians given a first invariant. J. Math. Phys. 1996, 37, 5509-5517. [CrossRef]

13. Ray, J.R.; Reid, J.L. Invariants for forced time-dependent oscillators and generalizations. Phys. Rev. A 1982, 26, 1042-1047. [CrossRef]

14. Günther, N.J.; Leach, P.G.L. Generalized invariants for the time-dependent harmonic oscillator. J. Math. Phys. 1977, 18, 572-576. [CrossRef]

15. Wolf, K.B.; On Time-Dependent Quadratic Quantum Hamiltonians. SIAM J. Appl. Math. 1981, 40, 419-431. [CrossRef]

16. Colegrave R.K.; Mannan, M.A. Invariants for the time-dependent Harmonic Oscillator. J. Math. Phys. 1988, 29, 1580-1587. [CrossRef]

17. Fernández Guasti, M.; Gil-Villegas, A. Orthogonal functions invariant for the time-dependent harmonic oscillator. Phys. Lett. A 2002, 292, 243-245. [CrossRef]

18. Fring, A.; Tenney, R. Time-independent approximations for time-dependent optical potentials. arXiv 2019, arXiv:1906.08840.

19. Moya-Cessa, H.; Guasti, M.F. Coherent states for the time dependent harmonic oscillator: The step function. Phys. Lett. A 2003, 311, 1-5. [CrossRef]

20. Fernández Guasti, M.; Moya-Cessa, H. Amplitude and phase representation of quantum invariants for the time-dependent harmonic oscillator. Phys. Rev. A 2003, 67, 063803. [CrossRef] 
21. Lewis, H.R.; Leach, P.G.L. Exact invariants for a class of time-dependent non-linear Hamiltonian-systems J. Math. Phys. 1982, 23, 165-175. [CrossRef]

22. Guedes, I. Solution of the Schrodinger equation for the time-dependent linear potential. Phys. Rev. A 2001, 63, 034102. [CrossRef]

23. Macedo D.X.; Guedes, I. Time-dependent coupled harmonic oscillators. J. Math. Phys. 2012, 53, 052101. [CrossRef]

24. Thylwe K.-E.; Korsch, H.J. The 'Ermakov-Lewis' invariants for coupled linear oscillators. J. Phys. A 1998, 31, L279-L285. [CrossRef]

25. Moya-Cessa, H.; Fernández Guasti, M. Time dependent quantum harmonic oscillator subject to a sudden change of mass: Continuous solution. Revista Mexicana De FíSica 2007, 53, 42-46.

26. Ramos-Prieto, I.; Espinosa-Zuñiga, A.; Fernández-Guasti, M.; Moya-Cessa, H.M. Quantum harmonic oscillator with time dependent mass. Mod. Phys. Lett. B 2018, 32, 1850235. [CrossRef]

27. Yeon, K.-H.; Kim, D.-H.; Um, C.-I.; George, T.F.; Pandey, L.N. Relations of canonical and unitary transformations for a general time-dependent quadratic Hamiltonian system. Phys. Rev. A 1997, 55, 4023-4029. [CrossRef]

28. Ray, J.R. Exact solution of the time-dependent Schrödinger equation. Phys. Rev. A 1982, 22, 729-733. [CrossRef]

29. Lewis, H.R. Classical and Quantum Systems with Time-Dependent Harmonic-Oscillator-Type Hamiltonians. Phys. Rev. Lett. 1967, 18, 510-513. [CrossRef]

30. de Ponte, M.A.; Santos, A.C. Adiabatic quantum games and phase-transition-like behavior between optimal strategies. Quantum Inf. Proc. 2018, 17, 149. [CrossRef]

31. Levy, A.; Kiely, A.; Muga, J.G.; Kosloff, R.; Torrontegui, E. Noise resistant quantum control using dynamical invariants. New J. Phys. 2018, 20, 025006. [CrossRef]

32. Barral, D.; Liñares, J. Quantum Light propagation in longitudinally inhomogeneous waveguides as a spatial Lewis-Ermakov physical invariance. Opt. Commun. 2016, 359, 61-65. [CrossRef]

33. Barral, D.; Liñares, J. Spatial propagation of quantum light states in longitudinally inhomogeneous waveguides. J. Opt. Soc. Am. B 2015, 32, 1993-2002. [CrossRef]

34. Perez-Leija, A.; Keil, R.; Moya-Cessa, H.; Szameit, A.; Christodoulides, D.N. Perfect transfer of path-entangled photons in Jx photonic lattices. Phys. Rev. A 2013, 87, 022303. [CrossRef]

35. Perez-Leija, A.; Hernandez-Herrejon, J.C.; Moya-Cessa, H.; Szameit, A.; Christodoulides, D.N. Generating photon encoded W states in multiport waveguide array systems. Phys. Rev. A 2013, 87, 013842. [CrossRef]

36. Fernández Guasti, M.; Moya-Cessa, H. Solution of the Schrödinger equation for time-dependent 1D harmonic oscillators using the orthogonal functions invariant. J. Phys. A 2003, 36, 2069-2076. [CrossRef]

37. Fernández Guasti, M. Energy content in linear mechanical systems with arbitrary time dependence. Phys. Lett. A 2018, 382, 3231-3237. [CrossRef]

38. Loudon, R.; Knight, P.L. Squeezed light. J. Mod. Opt. 1987, 34, 709-759. [CrossRef]

39. Yuen, H.P. Two-photon coherent states of the radiation field. Phys. Rev. A 1976, 13, 2226-2243. [CrossRef]

40. Caves, C.M. Quantum-mechanical noise in an interferometer. Phys. Rev. D 1981, 23, 1693-1708. [CrossRef]

41. Moya-Cessa, H.; Vidiella-Barranco, A. Interaction of squeezed states of light with two-level atoms. J. Mod. Opt. 1992, 39, 2481-2499. [CrossRef]

42. Barnett, S.M.; Beige, A.; Ekert, A.; Garraway, B.M.; Keitel, C.H.; Kendon, V.; Lein, M.; Milburn, G.J.; Moya-Cessa, H.M.; Murao, M.; et al. Journeys from quantum optics to quantum technology. Prog. Quantum Electron. 2017, 54, 19-45. [CrossRef]

(C) 2019 by the authors. Licensee MDPI, Basel, Switzerland. This article is an open access article distributed under the terms and conditions of the Creative Commons Attribution (CC BY) license (http:/ / creativecommons.org/licenses/by/4.0/). 